\title{
A Study on the Vibration Characteristics of EO/IR Gimbal for UAVs
}

Jae-Wook Choi , Researcher, Department of Aeronautical Systems Engineering, Hanseo University, Gomseom-ro 236-49 Nam-Myeon Trean-Gun Chuncheongnam-do,32158, Korea

*Dong-Gi Kwag, Professor, Department of Aeromechanical Engineering, Hanseo University, Gomseom-ro 236-49 Nam-Myeon Taean-Gun Chuncheongnam-do,32158, Korea,dgkwag@hanseo.ac.kr

${ }^{*}$ Corresponding Author

\begin{abstract}
The miniaturization of Unmanned Aerial Vehicle (UAV) and the resulting precision control technology have significantly grown in the modern world, and accordingly the development of smallsized surveillance equipment is urgently needed. This study aims to examine the vibration characteristics for the development of a 2-axis stabilization gimbal for small UAVs. Modal analysis and Harmonic response analysis were conducted to develop a 2-axis EO/IR gimbal with less than $300 \mathrm{~g}$. It examined the vibration characteristics of the gimbal in the range of $180 \mathrm{~Hz}$ to $720 \mathrm{~Hz}$, which is the vibration frequency band due to the propeller rotation of unmanned aerial vehicles, as well as the stability of the system. It analyzed the vibration characteristics of gimbal by using a commercial finite element program (Ansys Workbench). This study investigated the structural stability of the system by analyzing the resonance frequency and the vibration characteristics of gimbal frame, considering the vibrations from disturbances in UAVs. Modeling was carried out in consideration of the miniaturization and light weight of a 2-axis EO/IR Gimbal, and mesh optimization was performed by using Tetrahedron Mesh to improve the reliability of the vibration analysis result.
\end{abstract}

Keywords: Gimbal, Finite Element Method, Stabilization Design, Modal Analysis, Harmonic Analysis, Vibration Characteristics

Received: 07.12.2020 $\quad$ Accepted: 10.01.2021 $\quad$ Published: 05.02.2021

\section{INTRODUCTION}

As UAV technology has rapidly developed thanks to the development of miniaturization and precision control technology since the mid-2010 and is being widely applied in military/civil fields. With the rapid growth of military/civil UAVs, there is an urgent need to develop small surveillance equipment that acts as the eyes of UAVs. Particularly, in the field of defense, the importance of image information sensors and guided munitions that visualize the battlefield situation in real time are becoming increasingly important in the modern warfare. For the technology development of small surveillance equipment, this study was conducted to develop a 2-axis EO/IR (Electro-Optical/Infra-Red) gimbal with less than 300g for small UAVs.

The structure of gimbal systems can be largely divided into a camera module, a stabilization drive motor, and a frame. The camera module, which directly acquires an image, is composed of an EO optical system, an IR optical system, and an image processing board that processes the acquired image. The stabilization drive motor rotates with the Pan axis and the Tilt axis, with a built-in motor control board to perform control. The drive motor is used for constantly directing Gimbal's camera gaze or tracking a target. In addition, it plays a key role in minimizing the influence of external vibration and securing images by stably supporting the camera module. A gimbal that performs these functions is configured as shown in Fig. 1. For the stable use of a gimbal system, it is most desirable that there is no Natural frequency within a vibration frequency band. However, if the Natural frequency is structurally unavoidable, it should be in a low band that can achieve stabilization performance to optimize the vibration characteristics of the system. 


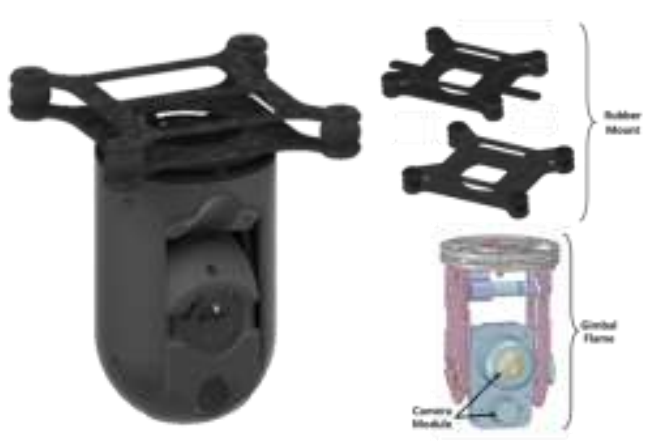

Figure 1 EO/IR Gimbal Shape

This study focused on analyzing the vibration characteristics in the range of $180 \mathrm{~Hz} \sim 720 \mathrm{~Hz}$, which is the vibration frequency band due to the propeller rotation among disturbances that are always applied to a gimbal during operation even in such a vibration disturbance environment. In addition, it analyzed the deformation shape and the frequency response function at the Natural frequency of a gimbal in a wide band of frequencies ranging from 1 to $2000 \mathrm{~Hz}$, through Ansys Workbench 20.0 in order to examine the shape of each mode.

\section{Analysis methods \\ 2.1 Modeling of EO/IR Gimbal}

In this study, the model to check the vibration characteristics is an EO/IR gimbal which is surveillance equipment equipped with UAV's day and night vision camera. The resonance caused by the vibration disturbances that happen during the operation of UAVs is not only a critical obstacle to the target positioning accuracy of a gimbal camera and the camera's sight stability, but also a factor that hinders the stability of the system. Therefore, this study intends to identify the Natural frequency and the vibration characteristics of a gimbal through Modal analysis [1-4] and Harmonic response analysis [5-8].

A gimbal is largely composed of an EO optical system, an IR optical system, an image processing board, a control board, a drive motor, and a gimbal frame, but 3D modeling in the analysis was conducted by removing bolts, nuts, motor parts, etc. of the gimbal frame. The camera module has a very complex configuration but was designed to align the center of gravity to the center of a gimbal system for system control. Therefore, for a vibration analysis, the camera module was defined as the mass at the relevant location for simplification of the analysis. The finite element modeling of bearing that considers the inner ball contact of bearing applied to each axis has limitations, so it is assumed that it is an integrated part and is completely fixed by the gimbal system. With regard to the design requirements for gimbals, the transmission rate of a gimbal camera must be 3 or less in the frequency band of $0 \mathrm{~Hz} \sim 500 \mathrm{~Hz}$ [9]. The gimbal system applied to the analysis is shown in Fig. 2.

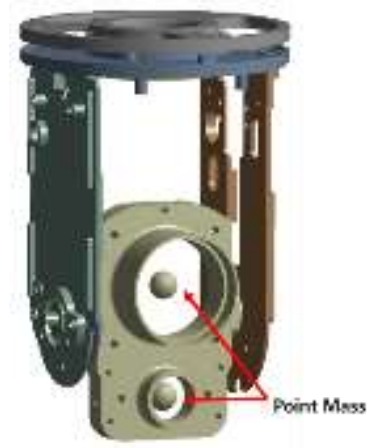

Figure 2 Analysis Shape of Gimbal

\subsection{Boundary Conditions}

The material used for gimbals should not only be rigid enough to withstand thermal deformation from heat and deformation from external force that may occur in a camera during the operation of UAV, but also light enough to not burden UAV[10]. To this end, the gimbal system was made of aluminum 6061 . 
In the case of the Mesh shape of an analysis model, Tetrahedrons Method was used as shown as Fig.3, which is composed of 249,459 material points and 138,969 elements. Through the Mesh analysis, the average element quality was formed to be about 0.7 , and the standard deviation of this Mesh was optimized to have 0.17. If the Mesh optimization is not considered, the result of the analysis will have an error which reduces the accuracy of the analysis and significantly lowers the reliability of the result value.

A 2-axis gimbal is greatly exposed to vertical excitation through the gimbal mounting surface in the installation direction. This study aimed to look into the characteristics of a 2 -axix gimbal in terms of disturbances that may occur during the operation by giving the input value for the vertical direction to the gimbal mounting surface and analyzing the vibrations. The disturbance conditions for the analysis are shown in Fig. 4.

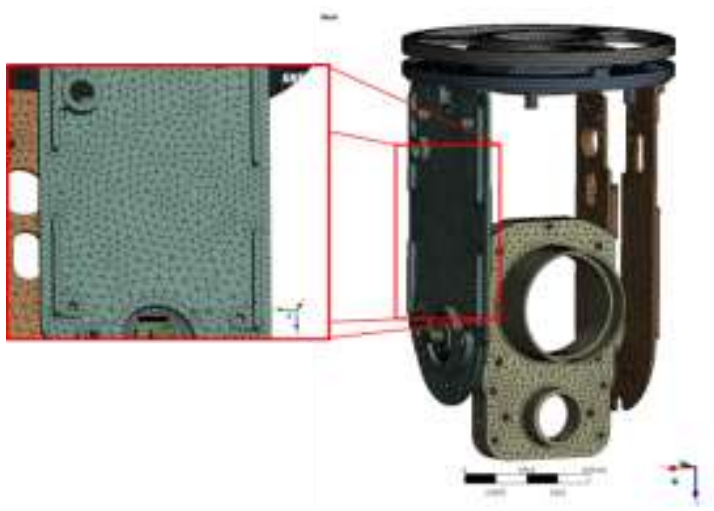

Figure 3 Mesh Shape of Gimbal

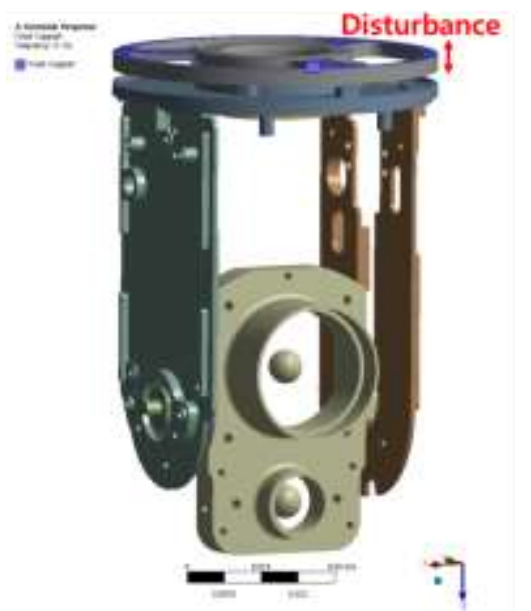

Figure 4 Disturbance Condition of Gimbal

\subsection{Rubber Mount Analysis}

Additional vibration reduction devices are needed to attenuate the vibration caused by the disturbance transmitted from the frequency band of interest to the gimbal. In this section, vibration analysis is used to determine the properties of the rubber mount. In the case of rubber mounts, due to the characteristics of rubber, the internal resistance due to viscosity is high, so the amplitude of vibration is not high even in the resonance region, and it has excellent vibration insulation performance in the high frequency nonresonant frequency band. In addition, it is easy to reduce size and weight, so it is advantageous to apply to the EO/IR gimbal of this study. The rubber mount was composed of upper and lower plates using AKSACA's Carbon Fiber, and in the case of rubber, U. S. Rubber's nitride rubber polymer material was used. The analysis was carried out by applying Base Excitation conditions to the drone mounting surface as in Section 2.2. The disturbance conditions are shown in Fig. 5. The rubber of the mount utilized the spring connection condition to simplify the analysis. The rubber damping ratio was assumed to have a value of $2.6 \%$ through experiments. Simplified modeling proceeded as shown in Fig.6. 


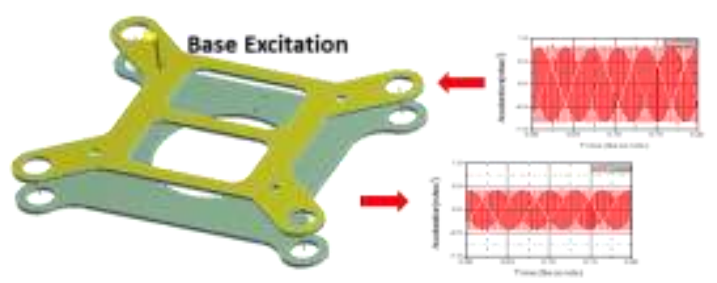

Figure 5 Disturbance Condition of Gimbal

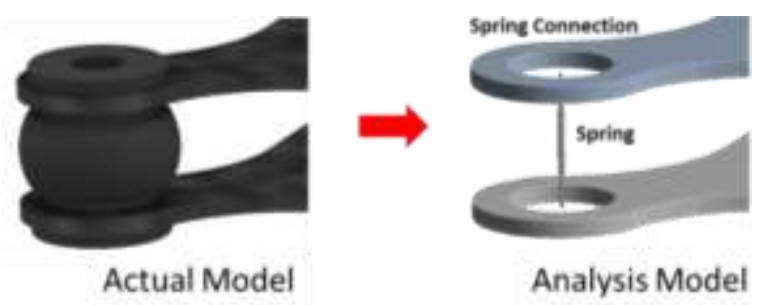

Figure 6 Simplification of Rubber

The mount shape consists of a general rubber mount model and a mount with a $130 \mathrm{~mm}$ long beam. Through analysis, the vibration reduction performance according to the presence or absence of a beam shape was confirmed. The mount model performed in the analysis is shown in Fig. 7.
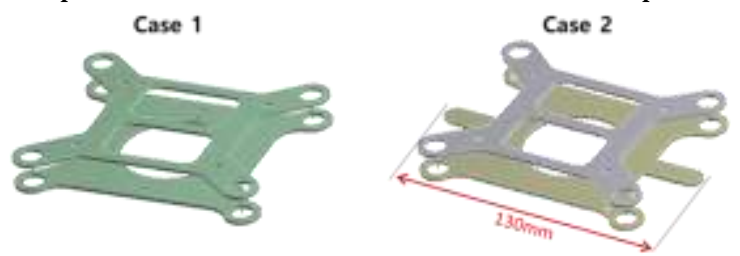

Figure 7 Analysis Shape of Rubber Mount

\section{Analysis Result}

3.1 Analysis Result of EO/IR Gimbal

Finite element analysis was performed to minimize resonance and Harmonic caused by external force applied to a gimbal system assumed to be rigid. Focusing on the vicinity of $180 \sim 720 \mathrm{~Hz}$, which is the frequency band of the propeller rotation center of UAVs, this study examined the characteristics of the band ranging from 0 to $2000 \mathrm{~Hz}$ and performed an analysis on whether a gimbal system was affected by vibrations. For the analysis, a commercial finite element program Ansys Workbench was used. In the case of FRF Data, the transmission rate was analyzed by receiving the acceleration data, given the assumption that a vibration disturbance is the acceleration input in the analysis. Data measurement point is shown in Fig. 8.

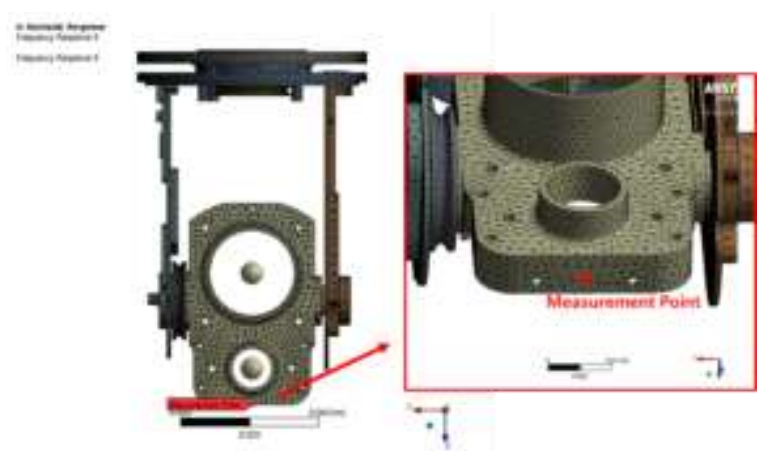

Figure 8 Measurement Point of Harmonic Analysis

The Mode shape of an E0/IR gimbal is shown in Fig. 12-14. The FRF (Frequency Response Function) is shown in Fig. 9-11 through the acceleration data of the $\mathrm{x}, \mathrm{y}$, and $\mathrm{z}$ axes obtained from the data measurement point in Fig. 8. In Fig. 9-11, (a) shows the transmission rate of $0 \sim 2000 \mathrm{~Hz}$ on a graph, and (b) shows the transmission rate of $180 \sim 720 \mathrm{~Hz}$, which is frequency band in this analysis, on an FRF graph. 


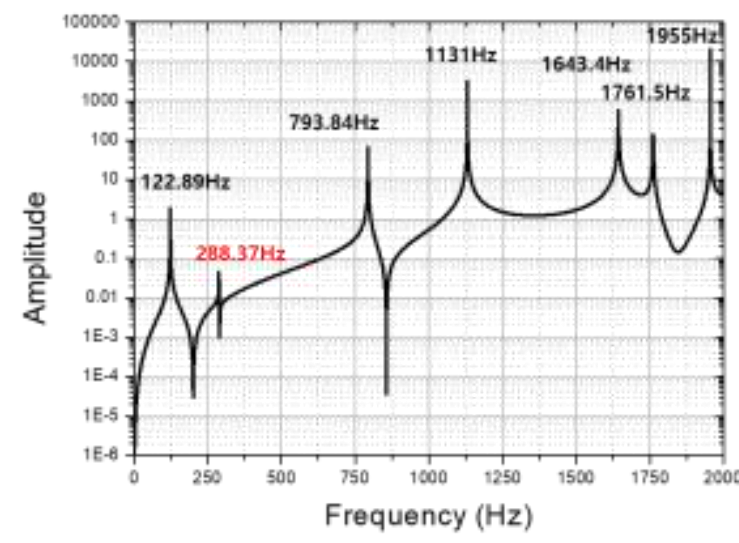

(a) FRF Graph $(0 \sim 2000 \mathrm{~Hz})$

Figure 9 FRF Graph of Gimbal of X Direction

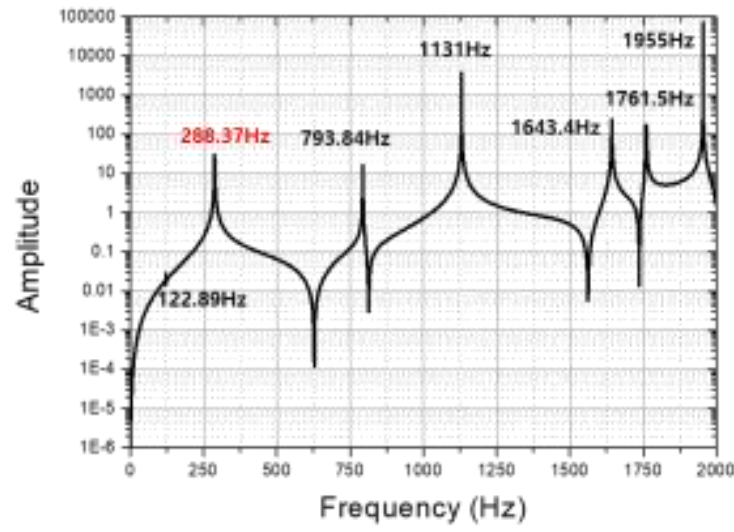

(a) FRF Graph (0 2000Hz)

Figure $10 \mathrm{FRF}$ Graph of Gimbal of Y Direction

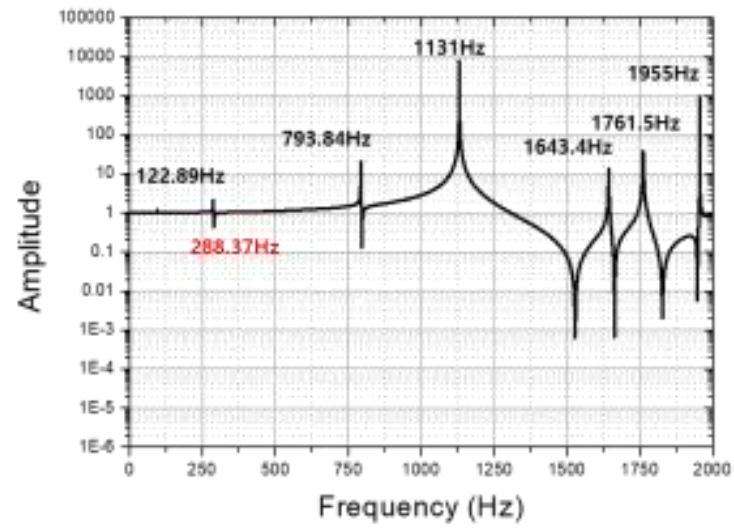

(a) FRF Graph (0 2000Hz)

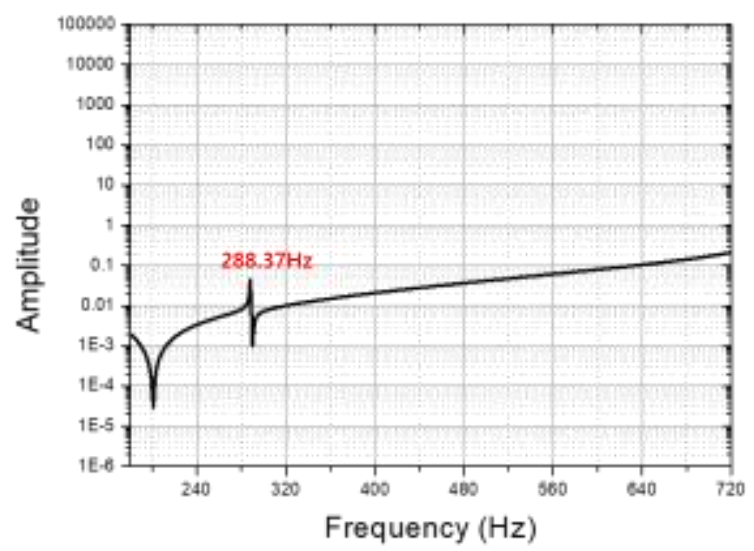

(b) FRF Graph (180 720Hz)

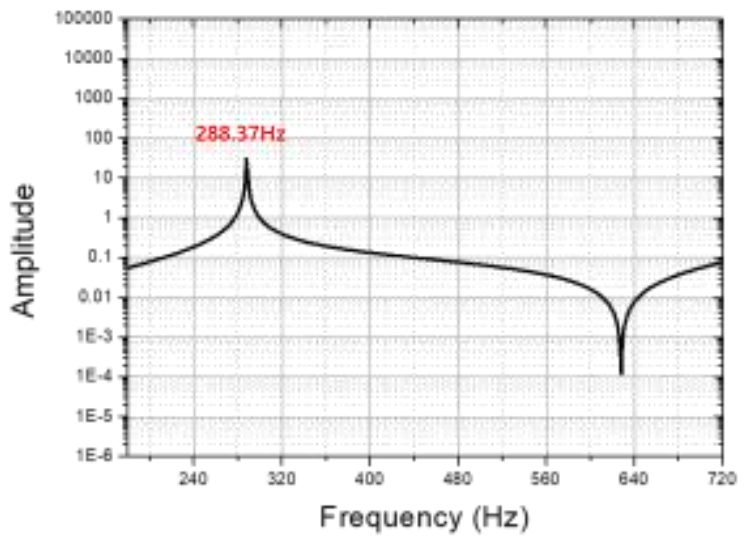

(b) FRF Graph $(180 \sim 720 \mathrm{~Hz})$

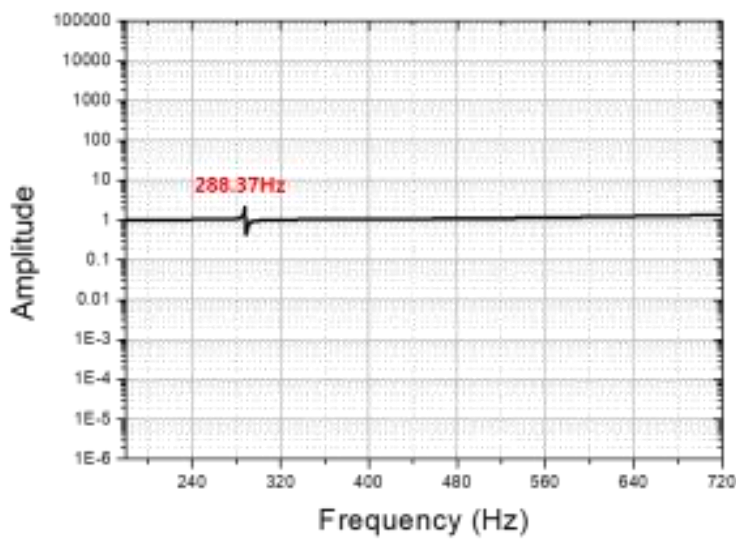

(b) FRF Graph (180 720Hz)

Figure 11 FRF Graph of Gimbal of Z Direction

The graph examined by the Harmonic response analysis shows that the maximum transmission rate of 1.88(x direction) occurs in the first mode $(122.89 \mathrm{~Hz})$, which falls within the range of the design requirements for gimbals. In addition, the FRF Graph in the frequency band of $180 \sim 720 \mathrm{~Hz}$ has found that there is one 1st Lateral Mode, and the maximum transmission rate is 30.11(y direction). 


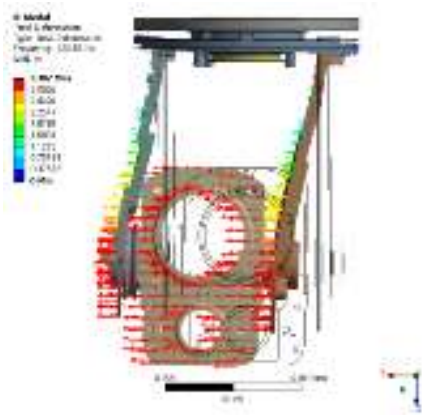

(a) $1^{\text {st }}$ Bending Mode $(122.89 \mathrm{~Hz})$

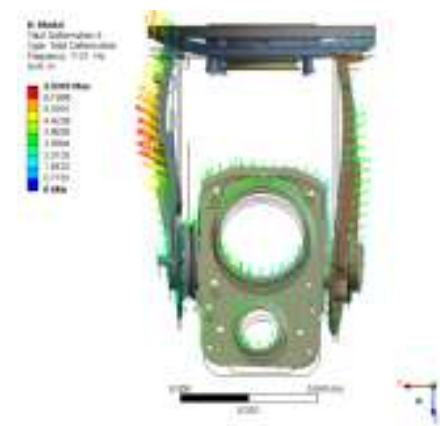

(b) $2^{\text {nd }}$ Bending Mode $(1131 \mathrm{~Hz})$

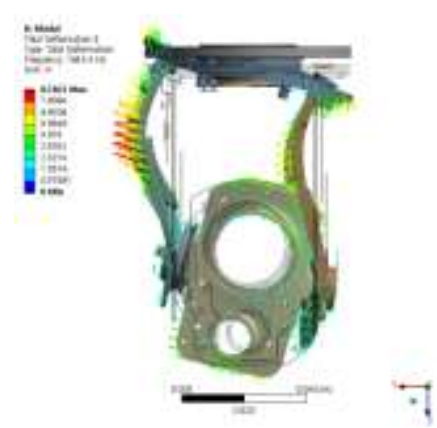

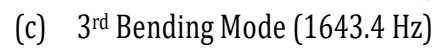

Figure 12 Bending Mode Shape of Gimbal

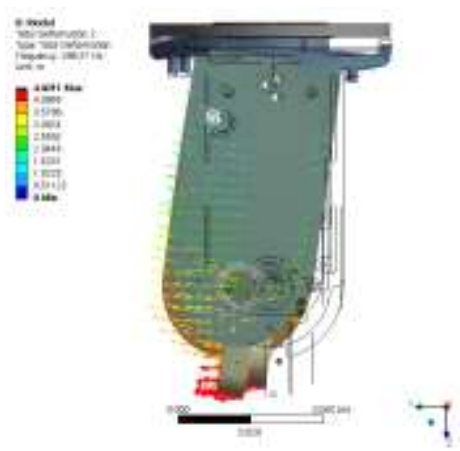

(a) $1^{\text {st }}$ Lateral Mode $(288.37 \mathrm{~Hz})$

Figure 13 Lateral Mode Shape of Gimbal

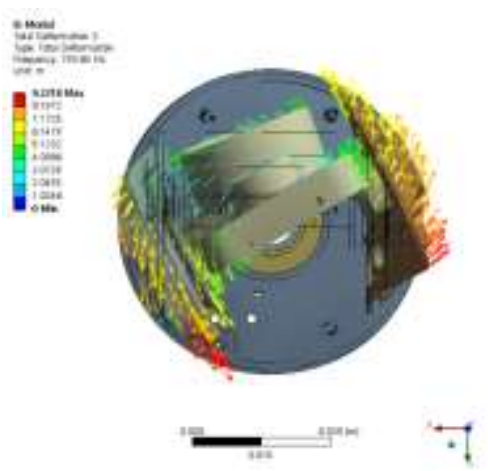

(a) $1^{\text {st }}$ Torsion Mode $(793.84 \mathrm{~Hz})$

Figure 14 Torsion Mode Shape of Gimbal

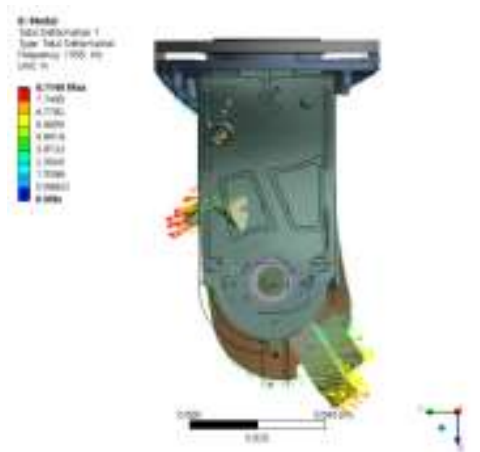

(b) $2^{\text {nd }}$ Lateral Mode $(1955 \mathrm{~Hz})$

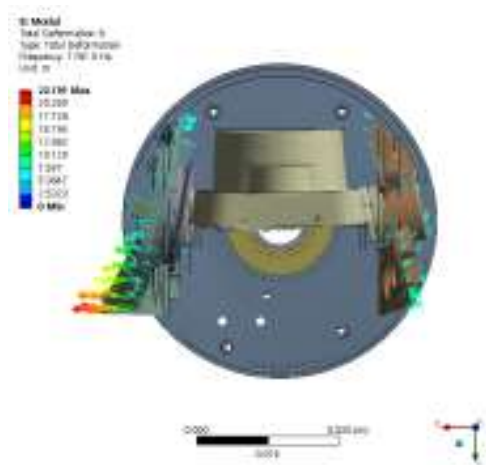

(b) $2^{\text {nd }}$ Torsion Mode $(1761.5 \mathrm{~Hz})$

In Fig. 12, (a), (b) and (c) are the 1st, 2nd, 3rd Bending Mode shape, and the Natural frequencies are 122.89Hz, 1131Hz, and 1643.4Hz, respectively. In Fig. 13, (a) and (b) are the 1st and the 2nd Lateral Mode shape, having Natural frequencies of $288.37 \mathrm{~Hz}$ and $1955 \mathrm{~Hz}$, respectively. In Fig. 14. (a) and (b) are the 1st and the 2nd Torsion Mode shape, and the Natural frequencies are $793.84 \mathrm{~Hz}$ and $1761.5 \mathrm{~Hz}$, respectively. 3.2 Analysis Result of Rubber Mount

In order to reduce vibration in the frequency band of interest, a study on rubber mounts was conducted, and the mount analysis has characteristics that are difficult to analyze due to the characteristics of viscoelastic material and carbon composite material at the same time. Harmonic response analysis and mode shape were checked in the range of 1 to $1000 \mathrm{~Hz}$ in order to confirm the characteristics in the range of 180 to $720 \mathrm{~Hz}$. In the case of the first natural frequency of the rubber mount, it occurs at $141.88 \mathrm{~Hz}$ and $151.25 \mathrm{~Hz}$ for each case and shows differences in high-frequency vibration characteristics depending on the presence or absence of a beam. FRF graph for each case confirmed through analysis through Fig. 15 is shown. The data of the FRF graph shows the Z-axis data applied to the gimbal according to the disturbance. In the frequency band of interest, the transmission rate is less than 1 and vibration is 
attenuated. Fig. 16 shows the mode shape of rubber mount.

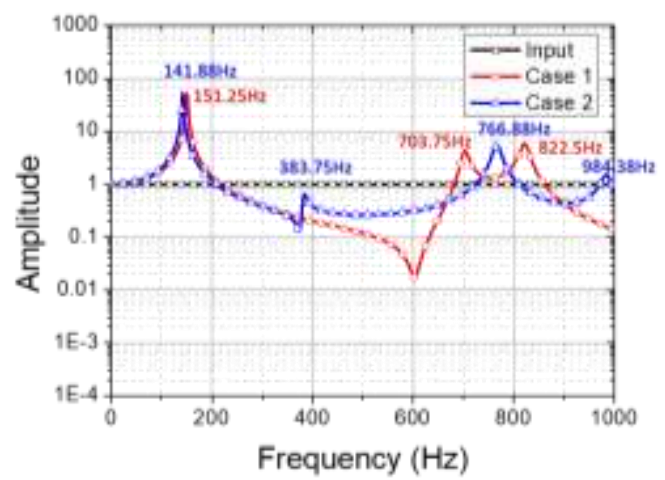

Figure 15 FRF Graph of Rubber Mount

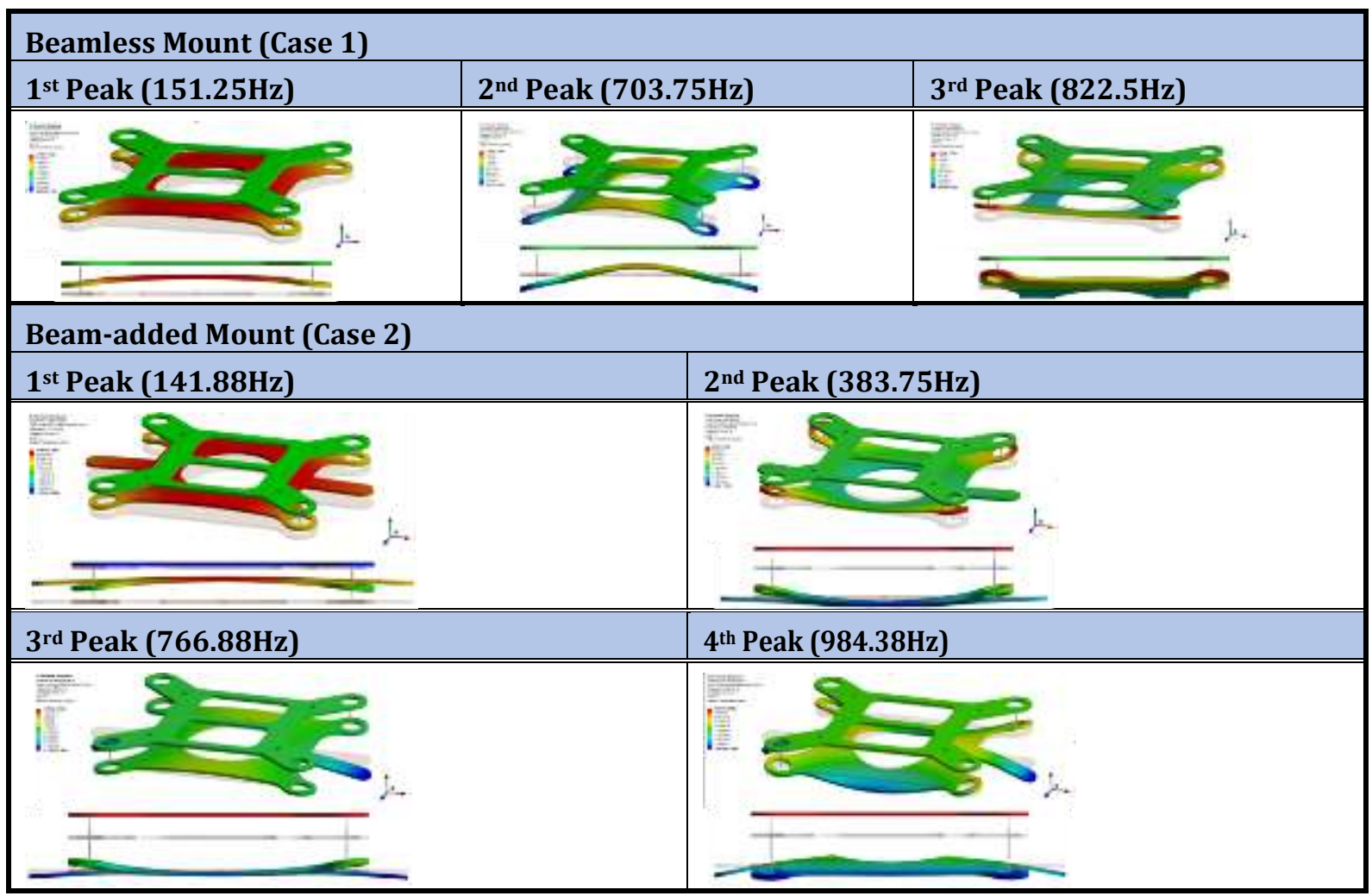

Figure 16 Mode Shape of Rubber Mount

\section{Conclusion}

A gimbal supports a camera module and performs image stabilization by applying stabilization control algorithms. In order to stably secure images and the accuracy of the camera shooting position, not only structural stability and strength but also the structural stabilization design should be achieved against external vibrations. This study verified that the system was designed to stabilize vibrations against resonance from disturbances. The vibration characteristics of an EO/IR gimbal were concluded as follows.

1) In UAV, there was only Natural frequency of the 1st Lateral Mode $(288.37 \mathrm{~Hz})$ within $180 \mathrm{~Hz} \sim 720 \mathrm{~Hz}$ which is the frequency band due to propeller rotation. The maximum trans -mission rate measured at the camera position in the Natural frequency was 30.11 which is a relatively big value.

2) Given that the natural frequency of the 1st Bending mode corresponding to the first Natural frequency in a gimbal is $122.89 \mathrm{~Hz}$, the gimbal system was design-ed to avoid the frequency band, and the transmission rate value of 1.88 was within the range of design requirements.

3) As for the gimbal analyzed in this study, the transmission rate at the 1st Bending mode falls within the design requirements, so it is thought to have achieved the structural stabilization design for the 1st Natural frequency.

4) A high transmission rate of 30.11 occurred in the 1st Lateral Mode. To solve this problem, it needs to 
additional-ly develop a vibration-proof structure for vibration reduction of gimbals. Further research on the design of vibration isolators for less vibrations will be helpful in designing vibration stabilization.

5) In order to minimize uncertainty about the data identified in this study, the actual vibration test is performed using test equipment in the future to compare and analyze the data.

\section{Acknowledgment}

This study is a research paper supported by the 2019 Graduate Student Research Fund Project from the Industry-Academic Coopera -tion Foundation, Hanseo University.

\section{References}

1. M. Chati, R. Rand, S. Mukerhjee. (1997) Modal analysis of a cracked beam. Journal of Sound and Vibrat ion, 207(2), 249-70.

2. G. Oliveto, A. Santini, E. Tripodi. (1997) Complex Modal Analysis of a Flexural Vibrating Be am with Viscous End Conditions. Journal of Sound and Vibration, 200(3), 327-45.

3. P. Verboven. (2002) Frequency-Domain System Identification for Modal Analysis. Doctoral dissertation. Vrije Universitet Brussel. Belgium.

4. G. Hearn, Rene. B. (1991) Modal Analysis for Damage Detection in Structures. Journal of American Society of Civil Engineer, 117, 3036-42.

5. Y. K. Ji, Y. S. Lee, (2002). Random Vibra-tion and Harmonic Response Analyses of Upper Guide Structure Assembly to Flow Induced Loads. Journal of the computation-al Structural Engineering Institute of Korea, 15(1), 59-68.

6. M. S. Kim, J. N. Kim, Y. S. Byun, J. Kim, B. S. Kang. (2016). Study on Analysis of Vibration Characteristics and Modal Test for a Quad-Rotor Drone. Journal of Korea Society for Precision Engineering, 33(9), 707-14

7. Daniel J. Inman (2013). Engineering Vibr-ation (Forth Edition). Location : Pearson.

8. Z. Fu, J. He (2001). Modal Analysis. Location : Elsevier.

9. S. E. Lee, T. W. Lee. (2011). Vibration Characteristic Analysis of Gimbal Structure System with Observ ation Reconnaissance Camera Module. Transactions of the Korean Society of Mechanical Engineers A, 35(4), 409-15.

10. S. E. Lee, T. W. Lee. (2010). Vibration Characteristic Analysis of Gimbal Structure in Collection Equipm ent of Image Information. Journal of the Korea Society Manufacturing Process Engineerings, 9(2), 20 5. 